This item was submitted to Loughborough's Research Repository by the author.

Items in Figshare are protected by copyright, with all rights reserved, unless otherwise indicated.

\title{
Analysis and comparison of different selenization routes for nanoparticle ink deposited Cu(In1-xGax)(SeyS1-y)2 solar cells
}

PLEASE CITE THE PUBLISHED VERSION

http://dx.doi.org/10.1109/PVSC.2016.7750014

PUBLISHER

(C) IEEE

VERSION

AM (Accepted Manuscript)

LICENCE

CC BY-NC-ND 4.0

\section{REPOSITORY RECORD}

Eeles, Alexander, Ali Abbas, Panagiota Arnou, Jake W. Bowers, Michael Walls, Stephen Whitelegg, Paul Kirkham, et al.. 2019. "Analysis and Comparison of Different Selenization Routes for Nanoparticle Ink Deposited Cu(in1-xgax)(seys1-y)2 Solar Cells". figshare. https://hdl.handle.net/2134/24053. 


\title{
Analysis and Comparison of Different Selenization Routes For Nanoparticle Ink Deposited $\mathrm{Cu}\left(\mathrm{In}_{1-\mathrm{x}} \mathrm{Ga}_{\mathrm{x}}\right)\left(\mathrm{Se}_{\mathrm{y}} \mathrm{S}_{1-\mathrm{y}}\right)_{2}$ Solar Cells
}

\author{
Alex Eeles ${ }^{1}$, Ali Abbas ${ }^{1}$, Panagiota Arnou ${ }^{1}$, Jake W Bowers ${ }^{1}$, John M Walls ${ }^{1}$, Stephen Whitelegg ${ }^{2}$, Paul \\ Kirkham ${ }^{2}$, Cary Allen ${ }^{2}$, Stuart Stubbs ${ }^{2}$, Zugang Liu $^{2}$, Ombretta Masala ${ }^{2}$, Christopher Newman ${ }^{2}$, Nigel \\ Pickett $^{2}$
}

\author{
1: CREST, Wolfson school, Loughborough University, Leicestershire, LE11 3TU, UK; 2: Nanoco \\ Technologies Ltd, Manchester, M13 9NT, UK
}

\begin{abstract}
This paper investigates the effect of using different selenization sources, namely elemental Se and $\mathrm{H}_{2} \mathrm{Se}$, on $\mathrm{Cu}\left(\mathrm{In}_{1-\mathrm{x}} \mathrm{Ga}_{\mathrm{x}}\right)\left(\mathrm{Se}_{\mathrm{y}} \mathrm{S}_{1-\mathrm{y}}\right)_{2}$ devices derived from depositions of nanoparticle inks. Nanoparticles used in this synthesis are chalcogenides (e.g. CuInGaS). The effect of the selenization species has a large effect on the performance and electrical properties of these devices. Elemental selenized devices show higher efficiencies $(>16 \%)$ compared to $\mathrm{H}_{2}$ Se processed devices $(<12 \%)$. Various techniques are used in this study, including Raman spectroscopy, TEM, I-V-T, EQE, admittance spectroscopy and $\mathrm{C}-\mathrm{V}$-T to identify the difference in performance between the two selenization methods. Differences are observed in both the bulk and interface properties of the devices.

Index terms-CIGS, nanoparticles, selenization, solution processing.
\end{abstract}

\section{INTRODUCTION}

Solution processed $\mathrm{Cu}\left(\mathrm{In}_{1-\mathrm{x}} \mathrm{Ga}_{\mathrm{x}}\right)\left(\mathrm{Se}_{\mathrm{y}} \mathrm{S}_{1-\mathrm{y}}\right)_{2}$ (CIGS) has attracted significant attention from both academic and industrial research due to advantages in material utilization, yield, throughput and cost reductions. Printing technology has the potential to significantly reduce the cost of CIGS PV module manufacturing by replacing vacuum deposition techniques. Another significant advantage of solution processing is that the ratio of the metals is fixed in the ink preparation and is independent of variation of the deposition thickness [1]. This is desirable for the deposition of films with uniform composition over a large area and may significantly help to close the efficiency gap between small area lab devices and production sized modules. In order to commercially realise the potential of solution processed devices it is essential to reach efficiencies similar to, or above, those achieved by vacuum processing methods.

The use of nanoparticle inks to print CIGS absorber layers has been previously employed, but many of the previous attempts use metal or metal oxide nanoparticles requiring significantly different selenization processes [2]. The implementation of chalcogenide nanoparticles capped with an organic ligand has already achieved $16.7 \%$ active area efficiency (13.6\% total area) and is expected to reach higher efficiencies in the near future [3]. Chalcogenide nanoparticles have significant advantages over metal or metal oxide nanoparticles because the chemical composition of the deposited films is close to the desired final composition. In contrast, metal oxide nanoparticles, for example, require an extra reducing step in $\mathrm{H}_{2} / \mathrm{N}_{2}$ gas [2] which is not necessary when using chalcogenide nanoparticle precursors. Additionally, commercial scale-up of chalcogenide nanoparticles has been opened up by the molecular seeding approach developed by Nanoco Technologies [4]. This technique allows low-cost, large scale production of a variety of high quality binary, ternary or quaternary chalcopyrite nanoparticles for use in nanoparticle inks.

After the deposition of the nanoparticle inks, a selenization step is performed to recrystallize the CIGS absorber layer. This selenization step is also widely used for vacuum deposited devices meaning industrial processes and supply chains are already developed. There are two different commonly available options for the selenization source: $\mathrm{H}_{2} \mathrm{Se}$ gas or elemental selenium (eSe). The most established method uses $\mathrm{H}_{2} \mathrm{Se}$, which achieves good results on large area devices $[5,6]$. However there are significant efforts to replace $\mathrm{H}_{2} \mathrm{Se}$ gas due to its high toxicity and difficult handling [7]. Thus investigations using both sources have revealed different advantages: one due to process maturity and the other due to reduced toxicity of chemicals used in the production line.

In this study, a significant efficiency gap exists between devices selenized using the elemental selenium source, with active area efficiencies over $16 \%$, and devices selenized using $\mathrm{H}_{2} \mathrm{Se}$ with $<12 \%$. This is despite similar grain size in the absorber of the finished films. This is the reverse of the usual finding from selenized metallic layers [1,8] and may be related to different chemical compositions of the selenized precursors. The purpose of the selenization step for these devices it to recrystallize the absorber layer to form large crystals. Fundamentally different chemical and physical processes may be occurring, compared to different manufacturing processes even though the desired end product is the same.

The purpose of this paper is to analyze the differences between devices fabricated using the different selenization 
routes to better understand the reason for the shortfall in efficiency when using the $\mathrm{H}_{2}$ Se selenization route.

Devices have been characterized using, Current-VoltageTemperature (I-V-T) analysis, external quantum efficiency (EQE) measurements, transition electron microscopy (TEM) imaging, admittance spectroscopy and Capacitance-VoltageTemperature (C-V-T). Raman spectroscopy was performed on the absorber films.

\section{EXPERIMENTAL DETAILS}

The nanoparticle ink was synthesized using Nanoco's patented molecular seeding approach to grow the nanopaticles, which were then coated with an organic ligand making them dispersible in common non-polar organic solvents. Different sized nanoparticles can be created and used. Fig. 1 shows images of 3nm and 20nm nanoparticles.

Devices were fabricated at Nanoco by spin coating the ink onto Mo coated glass followed by annealing in a $\mathrm{N}_{2}$ atmosphere to remove the solvent and ligand, which is then followed by subsequent selenization. After selenization, chemical bath deposition of the buffer layer, and sputtering of intrinsic oxide and TCO layers were conducted.

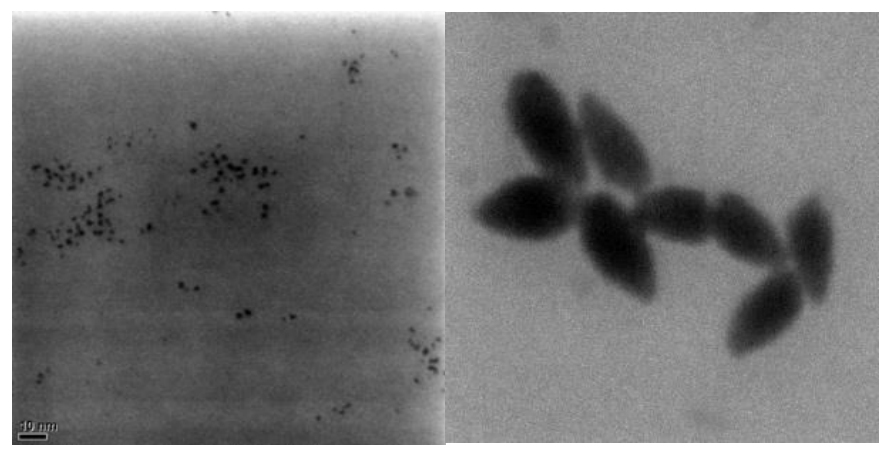

Fig. 1. Images of the nanoparticles. Left - 3nm nanoparticles. Right - 20nm particles.

Raman spectroscopy was performed using a Jobin Yvon JR confocal microscope with a 514nm excitation to investigate the presence of secondary phases, particularly the ordered defect complex (ODC). The Raman measurement was made after buffer deposition and subsequent removal. I-V-T measurements were performed using a liquid nitrogen cryostat, halogen light source and Keithley source-measurement unit. EQE measurements were made using a Bentham spectral response measurement system. Transmission electron microscopy (TEM) was carried out using a FEI Tecnai F20 (S)TEM equipped with a silicon drift detector, with an operating voltage of $200 \mathrm{kV}$. The TEM samples were prepared by Focused Ion Beam (FIB) milling and platinum was locally deposited as an over-layer. Admittance spectroscopy and C-V-
$\mathrm{T}$ measurements were performed using a liquid nitrogen cooled cryostat and Agilent E4980A LCR meter.

\section{RESULTS}

$\mathrm{J}-\mathrm{V}$ curves of the best performing devices are shown in Fig. 2. This shows how the efficiency of devices from the $\mathrm{H}_{2} \mathrm{Se}$ selenization process have lower open circuit voltage as well as short circuit current desnsity and fill factor.

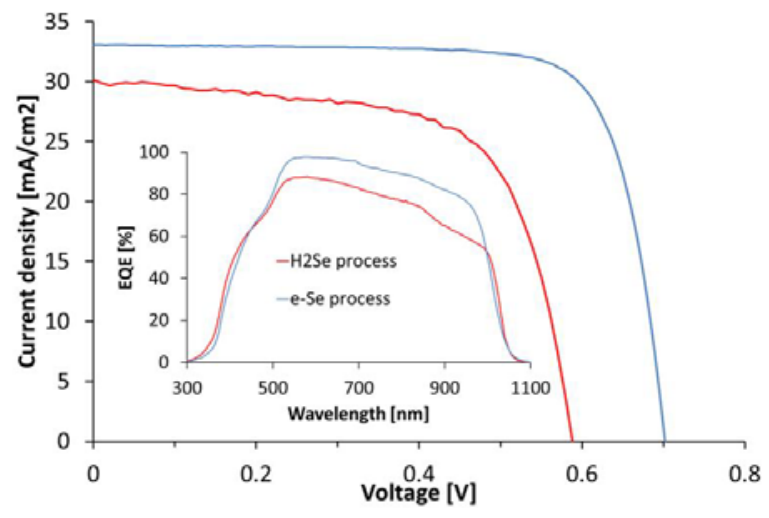

Fig. 2. Comparison of IV and EQE curves for devices obtained by each selenization route.

The bandgap of the devices was estimated using the maximum of the derivative of EQE measurements as $1.22 \mathrm{eV}$ for the $\mathrm{H}_{2}$ Se device and $1.20 \mathrm{eV}$ for the eSe device.

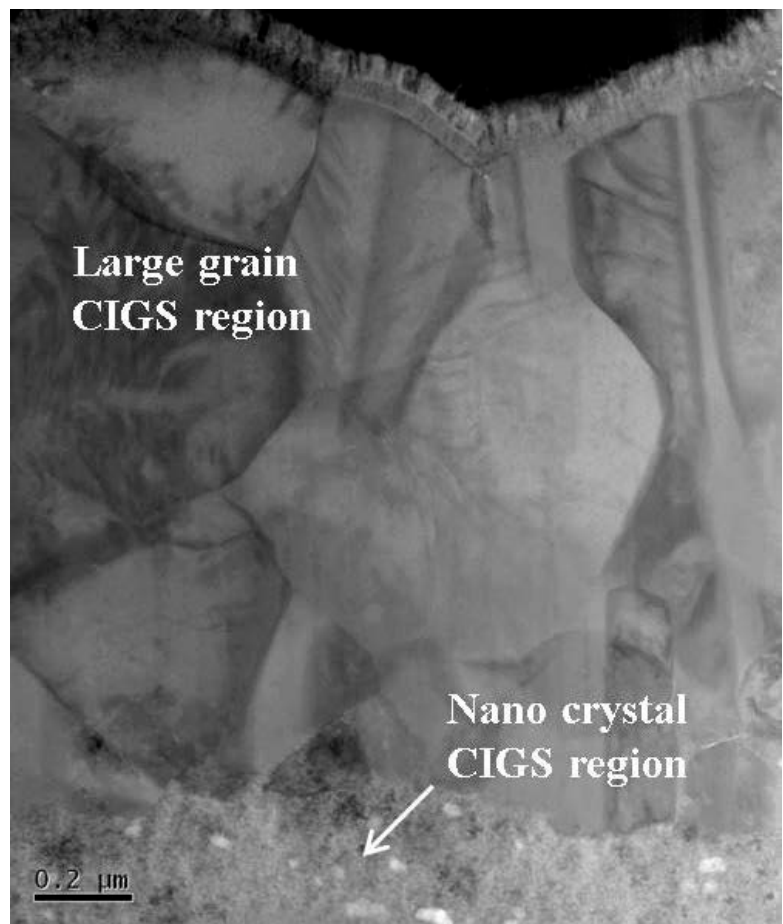

Fig. 3. TEM cross section showing the large and small crystal regions present in the device 
One particularity of the device created this way is that the CIGS forms in two layers distinguished by their grain size as observed in the TEM image shown in Fig. 3. The large grain region forming at the front and the smaller grain region at the back of the device. The relative size of these layers depends on the selenization conditions, such as time, temperature, or selenium vapour pressure. Similar grain sizes and widths of the small and large grain layers can be produced using either selenization species, so the difference in performance must have another origin.
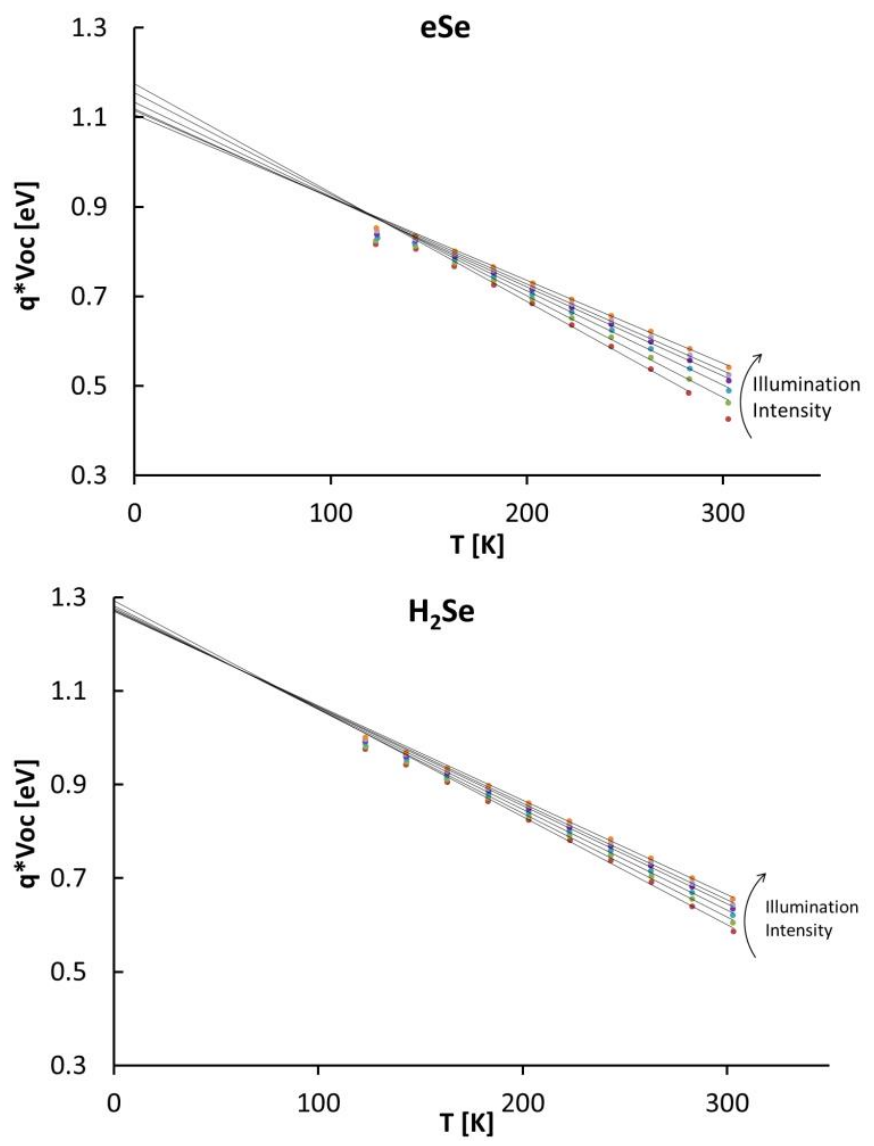

Fig. 4. $V_{o c}$ vs. temperature at different illuminations for each device type.

I-V-T data were taken in an effort to understand the limiting processes in the devices. Fig. 4 shows plots of the $\mathrm{V}_{\text {ос }}$ against temperature for devices selenized via each route. The intercept of the linear fit gives the activation energy for the dominant recombination mechanism. In most cases the activation energy should be equal to the bandgap at the position of the dominant recombination. However in the case of interface recombination and a pinned Fermi level at the interface, the activation energy will be smaller and is equal to the energy between the conduction band and the Fermi level at the interface [5]. The activation energy for recombination in the $\mathrm{H}_{2}$ Se devices is lower than that in the eSe device by about
$0.1 \mathrm{eV}$. However for the $\mathrm{H}_{2}$ Se device the measured activation energy is illumination dependant. At lower illuminations it agrees well with the bandgap but decreases by $0.1 \mathrm{eV}$ at 2 sun illumination. The activation is only $0.1 \mathrm{eV}$ lower than the bandgap, which might indicate interface recombination but is not a large enough difference to confidently make this interpretation.

The low temperature saturation of the $\mathrm{V}_{\mathrm{oc}}$ gives the maximum Fermi level splitting in the device [9]. The $\mathrm{V}_{\text {ос }}$ of the $\mathrm{H}_{2} \mathrm{Se}$ device starts to saturate at temperatures below $140 \mathrm{~K}$ to a value $<0.9 \mathrm{~V}$. In contrast, the saturation of $\mathrm{V}_{\text {ос }}$ of the eSe device cannot be observed in the investigated temperature range, but is in any case greater than $1 \mathrm{~V}$ and more than $0.1 \mathrm{eV}$ higher than the $\mathrm{H}_{2} \mathrm{Se}$ device. This indicates that at low temperatures the Fermi level in the $\mathrm{H}_{2}$ Se device is more than $0.2 \mathrm{eV}$ above the valence band. This is likely caused by insufficient p-type doping compared to the compensating donors and deep states.

Raman spectra were taken to investigate differences in the absorber surface. In particular Raman spectra are able to show the presence or otherwise of the ordered defect compound (ODC) and $\mathrm{Cu}_{2} \mathrm{Se}$ phases. The ODC layer is often observed on the surface of $\mathrm{Cu}$-poor CIGS devices and is reported to lower the valence band minima compared to the CIGS absorber layer, reducing the hole concentration at the interface with the buffer and hence reducing interfacial recombination [10]. Raman spectra of two representative devices are shown in Fig. 5 . The shoulder, observed near $150 \mathrm{~cm}^{-1}$, indicates the presence of the ODC phase at the surface of the eSe device [11], but absent from the $\mathrm{H}_{2} \mathrm{Se}$ device. This adds some support to the possibility that the lower activation energy in the $\mathrm{H}_{2}$ Se device is due to interface recombination. However, devices have also been fabricated via the elemental selenization route, which contain the ODC layer, but have a slightly reduced activation energy, similar to the $\mathrm{H}_{2} \mathrm{Se}$ device shown. Thus, the analysis of interface recombination is still in progress.

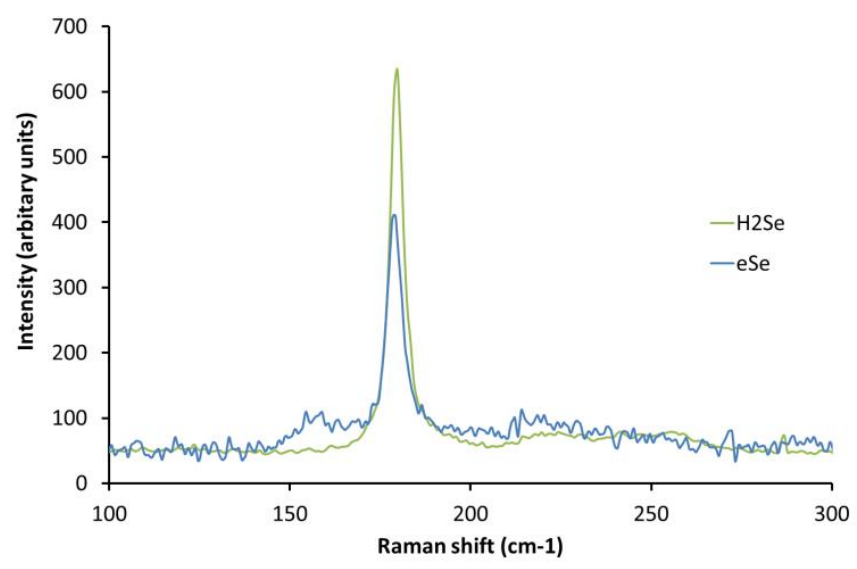

Fig. 5. Comparison of Raman spectra of samples selenized with $\mathrm{H}_{2} \mathrm{Se}$ and eSe. The peak close to $150 \mathrm{~cm}^{-1}$ shows the presence of the ODC complex at the surface of devices processed using eSe, whereas 
the absence of the same peak in the $\mathrm{H}_{2}$ Se device shows that the ODC layer is not present in that device.

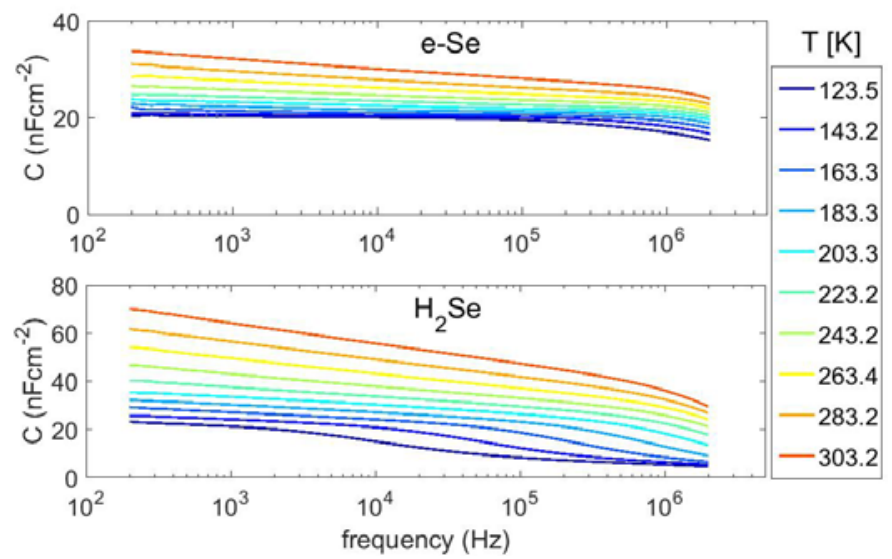

Fig 6: Admittance spectra of the devices.

Admittance and $\mathrm{C}-\mathrm{V}$ measurements were made to obtain more information about the electronic properties of the devices.

Fig 6 shows the admittance spectra of the devices. The $\mathrm{H}_{2} \mathrm{Se}$ device shows a large capacitance step that has a high frequency / low temperature limit of the capacitance equal to the expected geometric capacitance of the device, indicating carrier or mobility freeze-out [12]. From the I-V-T measurements (not shown), a very large increase in series resistance occurs at the temperatures as the capacitance step is observed. This is expected from a mobility freeze-out. Hopping conduction is observed in CIGS devices in the temperature range of these measurements, and may be the route cause of this capacitance step [13]. A characteristic of variable range hopping conduction is shown by the variation of conductance with frequency following a power law, $\mathrm{G}=A \omega^{s}$, where $\mathrm{A}$ and $\mathrm{s}$ are constants and $\mathrm{s}$ is between 0.8 and 0.9 . The measured conductance at $123 \mathrm{~K}$ fits excellently to this model with a value of $\mathrm{s}=0.82$ as shown in Fig 7.

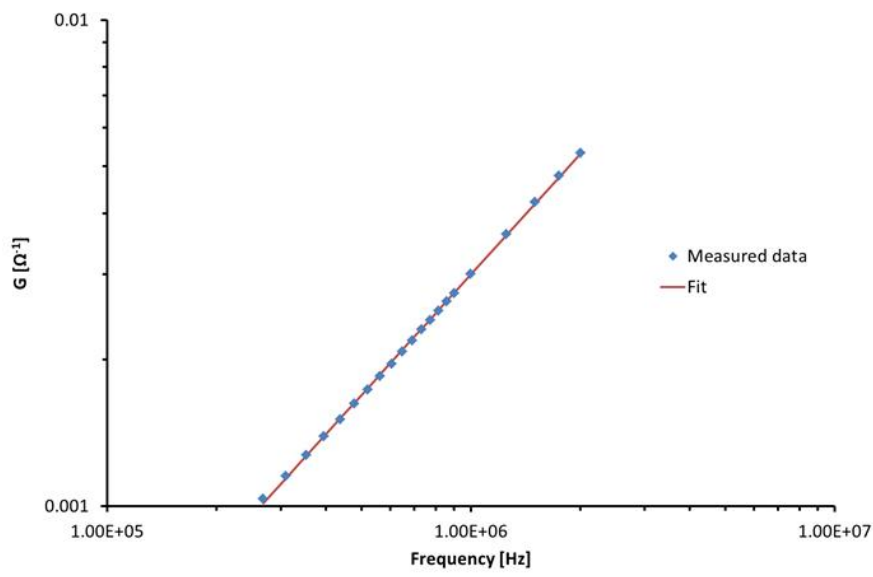

Fig 7: Conductance vs frequency at low temperatures showing the characteristic behaviour of variable range hopping conduction.
$\mathrm{C}-\mathrm{V}-\mathrm{T}$ measurements are shown in Fig 8. Provided the capacitance step corresponds to a mobility freeze-out, in order to calculate accurate doping densities, $\mathrm{C}-\mathrm{V}$ measurements should be taken above the step, i.e. before the capacitance starts to drop towards the geometric capacitance. However, since the step occurs at relatively high temperatures, it is possible trap states are influencing the extracted doping density measurement. In the case of the $\mathrm{H}_{2} \mathrm{Se}$ device, the extracted doping density continues to reduce substantially at temperatures down to the freeze out. Although the extracted doping density for the $\mathrm{H}_{2} \mathrm{Se}$ device appears higher than the eSe device, it is likely that this is an artificiality of the measurement because the maximum quasi Fermi level splitting suggests the doping in the $\mathrm{H}_{2} \mathrm{Se}$ device is much lower.

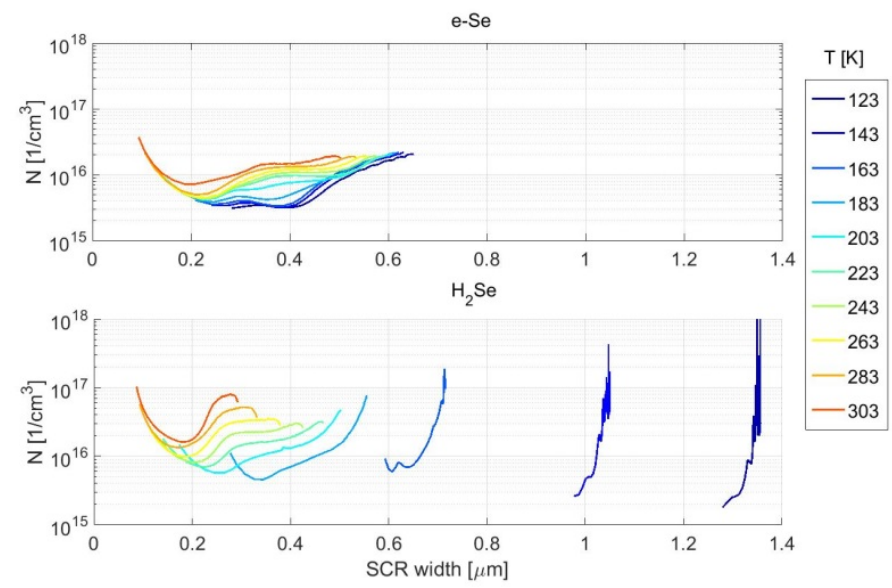

Fig 8: Doping density profiles from $\mathrm{C}-\mathrm{V}$ measurements at different temperatures.

\section{CONCLUSIONS}

The analysis suggests that there are several reasons for the lower performance of the $\mathrm{H}_{2} \mathrm{Se}$ device. Raman analysis showed the lack of an ODC layer in the $\mathrm{H}_{2} \mathrm{Se}$ devices. Along with slightly lower activation energy for recombination, interface recombination in the $\mathrm{H}_{2} \mathrm{Se}$ device is one possible cause. In addition, I-V-T, admittance and $\mathrm{C}-\mathrm{V}$ data suggest higher defect density and lower doping density in the $\mathrm{H}_{2} \mathrm{Se}$ device compared to e-Se device. Possible methods to improve the efficiency of the $\mathrm{H}_{2} \mathrm{Se}$ selenized device include methods to improve the doping such as sodium control and/or modification of the surface of the $\mathrm{H}_{2} \mathrm{Se}$ device to form the ODC layer observed in the elemental selenized device.

\section{ACKNOWLEDGEMENTS}

The authors would like to acknowledge the support of Innovate UK, project number 102235, and the EPSRC (EP/N508457/1) for the funding. We are also most grateful to Benjamin Bissig, Stephan Buecheler at EMPA for the use of 
their measurements system for the admittance, C-V-T and I$\mathrm{V}-\mathrm{T}$ and helpful discussions.

\section{REFERENCES}

[1] M. Kemell, M. Ritala and M. Leskela, "Thin film deposition methods for CulnSe(2) solar cells," Crit. Rev. Solid State Mat. Sci., vol. 30, pp. 1-31, 2005.

[2] V. K. Kapur, A. Bansal, P. Le and O. I. Asensio, "Non-vacuum processing of CuIn1-xGaxSe2 solar cells on rigid and flexible substrates using nanoparticle precursor inks," Thin Solid Films, vol. 431-432, pp. 53-57, 5/1, 2003.

[3] S. Whitelegg, P. Kirkham, C. Allen, S. Stubbs, Z. Liu, O. Masala and C. Newman, "Non-vacuum solution processed high efficiency CIGS PV devices," in EU PVSEC 2015, Hamburg, 2015,

[4] N. Pickett and J. Harris, "Preparation of nanoparticle material," jul \# 22, 2014.

[5] H. S. Roland Scheer, Chalcogenide Photovoltaics: Physics, Technologies, and Thin Film Devices. Wiley, 2011

[6] S. Niki, M. Contreras, I. Repins, M. Powalla, K. Kushiya, S. Ishizuka and K. Matsubara, "CIGS absorbers and processes," Prog Photovoltaics Res Appl, vol. 18, pp. 453-466, 2010.

[7] M. E. Erkan, S. D. Wu, C. Lee, M. Kim, D. Lim, K. Kim and M. H. -. Jin, "Chalcopyrite thin films and solar cells prepared by using selenoamide as a selenium source," Thin Solid Films, vol. 574, pp. 207-215, 1/1, 2015.

[8] J. Bekker, V. Alberts and M. Witcomb, "Influence of selenization techniques on the reaction kinetics of chalcopyrite thin films," Thin Solid Films, vol. 387, pp. 40-43, MAY 29, 2001.

[9] C. P. Thompson, S. Hegedus, W. Shafarman and D. Desai, "Temperature dependence of VOC in CdTe and $\mathrm{cu}(\mathrm{InGa})(\mathrm{SeS}) 2$-based solar cells," in Photovoltaic Specialists Conference, 2008. PVSC '08. 33rd IEEE, 2008, pp. 1-6

[10] D. Schmid, M. Ruckh, F. Grundwald and H. Schock, "Chalcopyrite Defect Chalcopyrite Heterojunctions on the Basis of Cuinse2," J. Appl. Phys., vol. 73, pp. 2902-2909, MAR 15, 1993.

[11] W. Witte, R. Kniese and M. Powalla, "Raman investigations of $\mathrm{Cu}(\mathrm{In}, \mathrm{Ga}) \mathrm{Se} 2$ thin films with various copper contents," Thin Solid Films, vol. 517, pp. 867-869, 11/28, 2008.

[12] J. T. Heath and P. Zabierowski, "Capacitance spectroscopy of thin-film solar cells," in Advanced Characterization Techniques for Thin Film Solar Cells, D. Abou-Ras, T. Kirchartz and U. Rau, Eds. Wiley, 2011, pp. 81-81-105

[13] U. Reislöhner, H. Metzner and C. Ronning, "Hopping conduction observed in thermal admittance spectroscopy," Phys. Rev. Lett., vol. 104, 2010. 